\title{
Ankara Oncology Training and Research Hospital
}

\author{
Guldeniz Argun \\ Additional information is available at the end of the chapter \\ http://dx.doi.org/10.5772/intechopen.76366
}

\begin{abstract}
Hip fracture is usually seen in advanced age. Due to the presence of metastases in elderly patients, the density of bones decreases, and the possibility of fracture increases. Cytokines, released from osteoclasts resulting in post-bony destruction, cause pain by activating pain receptors; hence, pain-related motion restriction may occur. Bone marrow suppression and medulla spinalis pressure may be seen in metastatic bone tumors. Three major complications can be observed in hip fracture operations: (1) perioperative bleeding, (2) bone cementum implantation syndrome and (3) thromboembolism. These indicate superiority of general anesthesia: 1 -controlled respiration. 2 -Invasive interventions to be performed during shock treatment can be made more easily and smoothly. 3 - The safest way to ensure that patients remain immobilized on the operating table. 4 Hemodynamic parameters can be better controlled. Regional anesthesia has benefits such as allowing early mobilization and postoperative pain control and few complications such as hypoxia, deep vein thrombosis and consciousness blurring. Various regional anesthesia methods such as spinal, epidural, combined spinal-epidural and peripheral nerve blocks are applied. The knowledge and experience of the anesthetist, the general condition and the mental state of the patient, the skill of the surgeon and the type of surgery determine the type of the anesthesia.
\end{abstract}

Keywords: hip fracture, regional anesthesia, general anesthesia

\section{Introduction}

Hip fracture is an important medical problem, which is seen in elderly patients, and has a higher incidence related to increased life expectancy, causing increased economic burden. Substantial increase of hip fractures is seen due to increased incidence of age-related osteoporosis and decreased balance resulted in falling. Fractures are more common in women, since osteoporosis is seen frequently in women. About $15-30 \%$ of patients with hip fracture 
die in a year. In addition to osteoporosis, hip fractures due to bone metastases are too high to be neglected. Current treatment is to provide patient weight bearing as soon as possible. Hemiarthroplasties and locked hip nails are the first surgical treatment methods [1].

The most common problems faced in clinical practice are as follows:

- Periosteum stretching

- Intramedullary hypoxia

- Infiltration and compression in the nerve roots

- Reflex muscle spasm

- Pathological fracture formation

- Direct bone invasion and destruction

- Pain due to activation of pain receptors by cytokines (prostaglandin, etc.), released by osteoclasts due to bone destruction

- Limitation of movement due to pain

- Bone marrow suppression due to medulla spinalis pressure and bone marrow infiltration

Non-surgical treatment is only available to patients with poor general conditions that are too low to tolerate any of the anesthetic methods. Hip fracture treatment requires a treatment choice, which needs to be individualized at the highest level. The choice of treatment should be based on surgeon, type of fracture, patient's age and health status. In an elderly patient population, hip fractures due to falling are among the first causes of death. An important factor determining the mortality in these surgeries is an advanced age; $30 \%$ of these cases are above 85 years of age.

Complications due to hip fracture operations:

- Bone cement implantation syndrome

- Perioperative bleeding

- Thromboembolism

\section{Bone cement}

Bone cement is a chemical substance consisting of methyl methacrylate polymers, which tightly adheres prosthetic material to the patient's bone by filling in the slots of the spongiosa. The exothermic reaction followed by the placement of the cement causes the cement to harden and expand. As a result, a pressure greater than $500 \mathrm{mmHg}$ is seen in the bone medullary. This pressure causes fat, bone marrow and air embolism in femoral deep veins. In addition, 
residual methyl methacrylate monomers cause vasodilatation and reduce systemic vascular resistance. Release of tissue thromboplastin triggers platelet aggregation that leads to micro thrombus formation in the lungs and cardiovascular instability due to vasoactive substances in circulation [2].

Clinical signs of complication related to bone cement are as follows:

- Hypoxia (increased pulmonary shunt).

- Hypotension.

- Arrhythmia (including heart block and sinus arrest).

- Pulmonary hypertension (increased pulmonary vascular resistance).

- Reduced cardiac output.

- Embolism most commonly occurs when a femoral prosthesis is placed.

Methods to reduce cement-related complications:

- Increasing oxygen concentration in inspiration before cement procedures

- Close monitoring of the patient's fluid balance

- Opening a hole in the distal region of the femur (vent hole)

- Cleaning the femoral shaft with high-pressure lavage to remove debris

- Using prosthesis without cement

\section{Bleeding}

Hip surgery is an operation causing significantly blood loss. Average loss of blood is about 1000-1500 ml. Bleeding cause depends on several factors. These factors are as follows:

- Experience of the surgeon.

- The surgical technique and the type of prosthesis.

- Controlled hypotension may reduce intraoperative bleeding.

- Some studies have shown that loss of blood in regional techniques (spinal and epidural) providing equal average blood pressure was 30-50\% less than general anesthesia [3, 4].

- For bleeding control, preoperative embolization, autologous and allogeneic transfusions and hemodilution methods may be applied. Aprotinin, epsilon aminocaproic acid and tranexamic acid prevent blood loss without increasing thrombus formation risk [5]. 


\section{Venous thromboembolism}

Factors that increase the risk of thromboembolism are as follows:

- Previous thromboembolism history

- Previous venous surgery and/or varicose surgery history

- Previous orthopedic operations

- Advanced age

- Malignancy

- Congestive heart failure

- Chronic lower limb laceration

- Immobilization

- Obesity

- Oral contraceptive and estrogen

- Unnecessary blood transfusions

Regional anesthesia reduces the incidence of thrombus formation and pulmonary embolism [6]. Davis et al. found that the deep vein thrombosis was $13 \%$ in spinal anesthesia, while $27 \%$ in general anesthesia [7]. Other approaches that reduce the risk of thromboembolism are the use of devices providing intermittent compression to legs and low-dose anticoagulant prophylaxis.

Pulmonary artery and its branches are obstructed by venous thrombus from systemic veins. There is a close relationship between pulmonary embolism (PE) and deep vein thrombosis (DVT). DTV is responsible for $90 \%$ PE. In 1856, Wirchow described clinical triad about the reasons of venous thromboembolism:

- Venous stasis

- Hypercoagulopathy

- Local injury at vessel walls (intraluminal damage)

Clinical impact of PE depends on several factors:

- Width of obstructed vessels bed

- Humoral factors released by serotonin and thromboxane A2

- Whether the patient has cardiopulmonary disorders or not

- Patient's age and general health status 


\subsection{Diagnosis}

- Chest X-ray

- ECG

- Arterial blood gas analyses:

- Major findings include hypoxemia, hypocapnia, respiratory alkalosis, and alveolar-arterial gradient increase $\left(\mathrm{PO}_{2} \mathrm{~A}-\mathrm{a}\right)$.

- Biochemical investigations: While CPK-MB, D-dimer, FDP, SGOT are normal, LDH and bilirubin are high.

- Respiratory function tests:

- The physiological dead space (VD) and the tidal volumetric ratio (VD/VT) are increased.

\subsection{Treatment}

- Ventilation with $100 \% \mathrm{O}_{2}$.

- Heparinization.

- Inotropic agents.

- Surgical embolectomy is performed in the large embolus. The small embolus is anticoagulated. The best treatment is prophylaxis.

- Venous stasis should be avoided. For this purpose, the legs should be lifted up and early mobilization should be provided postoperatively. Advanced venous stasis causes deep vein thrombosis and pulmonary embolism.

\section{Venous air embolism}

Low venous pressure and open ventilation are required for the air to enter the venous system as compared with the atmospheric pressure.

This most often occurs during the neurosurgery operations in a sitting position.

In addition, poor surgical technique may increase the formation of air embolism.

Factors affecting air embolization are as follows:

- Intravascular air volume

- Air inlet velocity

- The use of nitrogen protoxide

- Foramen ovale presence

- General health status 
Slowly entering a small air bubble has usually minimal physiological importance. In this case, venous air bubbles are taken out from lungs based on increased compensator pulmonary artery pressure (PAP). If this situation exceeds the capacity of lung removal, PAP increases, cardiac output (CO) decreases and hearth failure occurs. If balanced, namely, if venous air input is equal to pulmonary removal, PAP reaches a plateau.

$300-\mathrm{ml}$ air is fatal in adults. In animal trials, $1 \mathrm{ml} / \mathrm{kg} / \mathrm{min}$ causes embolism signs; $3-8 \mathrm{ml} / \mathrm{kg}$ causes death. The earliest sign is cardiovascular collapse. Blood pressure decreases dramatically; sudden hypotension, tachycardia, arrhythmia and cardiac arrest occur in succession. CVP increases; a metallic sound is heard with the precordial or esophageal stethoscope. In case of this, characteristic "sound of the mill wheel" is heard. This sound can be heard in all precordium. Respiratory changes such as increased respiratory rate, irregularity and apnea can be seen.

\subsection{Diagnosis}

- Doppler ultrasonic flow meter

- PAP

- End-tidal $\mathrm{CO}_{2}$

- Other methods: Monitoring should be done for patient in a sitting position. In these patients, continuous measurement of arterial blood pressure, $\mathrm{CVP}$, end-tidal $\mathrm{CO}_{2}$, Doppler ultrasound and esophageal stethoscope should be applied.

\section{Fat embolism}

\subsection{Etiology}

- Bone fractures

- Liver and kidney ruptures

- Rarely, after bone surgery

\subsection{Diagnosis}

With the presence of free fat in sputum and urine and in histological examination, petechiae and hyperlipidemia are diagnosed, with maximum level of free fat being reached in 3-4 days [8].

\subsection{Treatment}

Hypovolemia and shock should be treated. Clofibrate (atromid-S) and alcohol should be used for hyperlipidemia. 


\subsection{Preanesthesia assessment in arthroplasties}

- Personal background.

- Physical examination:

- The patient's age, weight, body mass index and vital signs (blood pressure, heart rate, pulse rate, body temperature, number of breaths, (depth breathing)) of the patient are evaluated.

- In these patients, the underlying etiology is mostly osteoarthritis or rheumatoid arthritis. Therefore, examination of the cervical and lumbar vertebrae is especially important for anesthesia.

- The cervical spine mobility should be checked to evaluate any restriction and assessed for intubation difficulty. Also, under the anesthesia and during intubation, attempts should be avoided from excessive extension of the neck joint.

- Osteoarthritis may lead to nerve root compression in the spine. Cervical spine and temporomandibular joint may be involved in patients with rheumatoid arthritis. In the presence of atlantoaxial joint subluxation, diagnosis can be made radiologically. During intubation, subluxation of the atlantoaxial joint may cause foramen magnum protrusion to odontoid process, reducing vertebral blood flow and compressing the spinal cord or brain. Intubation must be done by stabilizing the neck. A vigilant intubation technique may be preferred in some patients. It may be easier to prefer regional anesthesia in this group of patients [9].

- Because of multiple joint involvements in rheumatoid arthritis, it should be taken into account when placing intravenous catheters to the hand, wrist, foot, etc.

- Since most of patients undergoing arthroplasty are older, having cardiac, respiratory, cerebral, renal and endocrine diseases, perioperative and postoperative morbidity and mortality increase. Therefore, evaluation of systems is very important.

\subsubsection{The evaluation of cardiovascular system}

MET's (classification of metabolic equivalent) meaning is that 40 years old, $70-\mathrm{kg}$ man expresses oxygen consumption at rest. It is considered to be over 4 in the MET evaluation, can go uphill, go up 2 stories of stairs, walk straight on the road at $6 \mathrm{~km} / \mathrm{h}$, run short distance, do heavy work at home and participate in moderate activities. Patients with MET $\geq 4$ are considered to be good in terms of functional capacity.

- 1 MET resting, eating, toilet needs

- 3 MET mild housework, walking at 2-3 mph

- 4 MET 2-story climbing stairs 
- 7 MET heavy housework

- $\geq 10$ MET heavy sports ability

6.4.2. Cardiovascular risk assessment: the Lee risk index is the most appropriate for orthopedic patients

Number of current risks and incidence of cardiac complications

- $00.4 \%$

- $10.9 \%$

- $27 \%$

- $>311 \%$

\subsubsection{Cardiovascular drug management}

- Antihypertensive agents should not be discontinued until morning of the operation.

- Beta blockers should not be discontinued until an operation; the drug dose should be adjusted so that the heart rate is below $65 / \mathrm{min}$.

- Aspirin reduces the risk of deep vein thrombosis and pulmonary embolism. The use of aspirin alone provides suboptimal protection for thromboembolic events associated with hip fractures. It is imperative that aspirin should be continued for stapled, drug-free stents.

- Thienopyridine (clopidogrel and ticlopidine) combined with dual use of aspirin is associated with the use of coronary stent. Dual use increases the possibility of bleeding by $0.4-1.0 \%$ compared with aspirin treatment alone. Three ways may be followed based on the type of stent and intervention time:

- Dual treatment is continued without interruption.

- Bridging therapy with antithrombotic agents (low molecular weight heparin, glycoprotein II b/III a inhibitors) can be applied.

- Thienopyridine treatment is discontinued 10 days before surgery and resumed in a safe period.

Antithrombotic agents; fondaparinux, unfractionated heparin (UFH) and low molecular weight heparin (LMWH).

\section{Airway assessment}

There is a risk of difficult mask ventilation and difficult intubation due to hardness in the neck, dental deterioration and cervical joint degeneration. 
Hypoxia may be associated with pulmonary collapse and/or infection. Preoperative physiotherapy and spirometer may be beneficial to postoperative hypoxia.

Death, pneumonia, prolonged extubation and persistent bronchospasm can be seen in patients with COPD and smokers. The length of stay in intensive care unit is longer. The incidence of postoperative pulmonary complications is about $25-90 \%$ (If FEV1 $<65 \%$, risk of complications $>50 \%$ ). Arterial blood gas analysis may be done. If the value of $\mathrm{PaCO}_{2}$ is above $45 \mathrm{mmHg}$, it is a strong risk factor for preoperative pulmonary complication. In case of preoperative inhaler use, respiratory exacerbations and the presence of lung infection, antibiotic and supportive treatment should be provided with optimal conditions.

\section{Neurological assessment}

In the group of patients planned for arthroplasty, weakening of the memory, decrease in cognitive and intellectual functions, diminished movements, deterioration of sleep order, decrease in visual, acoustical, taste and smell sensation, autonomic nervous system imbalances, Parkinson, depression and dementia are common. If there is a suspicion of a mental condition of the patient, the mental status test (Mini Mental Status Test) can be planned.

\section{Renal assessment}

There is a risk of renal insufficiency and preoperative azotaemia related to age. Prerenal azotaemia should be corrected by hydration, by taking patients into dialysis program if necessary and by optimizing biochemical values.

\section{Glycaemia control}

Autonomic dysfunction, which is usually caused by diabetes mellitus in a long term, is an important actor for regional anesthesia and for control of perioperative hemodynamic. There is a possibility of silent myocardial ischemia. Oral antidiabetics should be stopped at least 24 hours before the operation.

\section{Nutrition control}

Because patients undergoing arthroplasty are geriatrics, preoperative nutritional status should be evaluated. Their nutritional status is assessed by Mini Nutritional Assessment Test. If albumin is $<3.5 \mathrm{gr} / \mathrm{dl}$, the patient should be supported. Reduced glomerular filtration rates due to age should be considered. Urea, serum creatinine and electrolytes should be examined. 


\section{Pain education}

Preoperative training including surgical procedure and rehabilitation protocol, informing the prospective benefits of the procedure, postoperative pain level and pain management techniques (intravenous, epidural patient-controlled analgesia), is beneficial to reduce patient anxieties, increase patient satisfaction, and reduce rehabilitation and hospital stay. Pre-emptive administration of opioid analgesics, NSAII and COX-2 inhibitors can provide effective postoperative analgesia. However, it should be considered that opioid use is associated with increased postoperative nausea and vomiting risk. Pre-emptive epidural analgesia with an epidural catheter prior to arthroplasty can provide postoperative pain control at a significant level [10].

\section{Approaches to oncological patients}

Systematic evaluation of oncologic patients is important. Patients are questioned about whether they have received chemotherapy (RT) or radiotherapy (RT). In these patients, impaired health status and blood vessel changes due to bone marrow suppression may be seen.

Alkylating agents such as cyclophosphamide may cause prolongation of the neuromuscular block.

It should be kept in mind that oxygen therapy at high concentration increases interstitial pneumonia and interstitial fibrosis due to bleomycin.

Skin reactions related to RT and KT should be noted. Vascular access may be problematic. Most cancer patients are malnourished due to their illnesses and side effects of treatment. Loss of appetite, cachexia, hyperproteinemia and hypoalbuminemia are common. Preoperative nutritional support should be given. Immunonutrition is thought to be useful to reduce immunosuppression. Lung metastases may be found to impair respiratory functions. The cardiotoxic effects of KT and RT may extend to cardiomyopathy, pericardial effusion, pericarditis and congestive heart failure.

In the hepatobiliary system, hepatomegaly, deterioration in liver function tests and even cirrhosis can be seen. Decrease in coagulation factors and coagulopathy can be seen.

In the urinary system, radiation nephropathy can be seen.

In the hematopoietic system, immune system impairment may be seen.

Multidisciplinary examination of cancer patients should be done. After their clinical and laboratory values are improved, the patients should be given to surgery.

\section{Anesthesia techniques in hip fracture operations}

\subsection{General anesthesia}

- It may be possible for hip fracture operations to obtain safer results with general anesthesia. 
- With controlled breathing, it may be possible to obtain the patient's alveolar oxygen pressure to the levels that meet the oxygen needs of the tissues.

- Invasive interventions to be done for the treatment of shock and monitoring of unconscious patient can be completed more easily and free of problems.

- It is the safest way for patients with restless and agitation due to shock and pain due to trauma to remain immobilized on the operating table.

- General anesthesia has some advantages (better control of hemodynamic parameters, given oxygen with higher concentration compared to regional anesthesia) [11].

Important points in general anesthesia:

- Firstly, enough volume should be replaced.

- Induction under appropriate monitoring conditions following a good pre-oxygenation to reduce hypoxemia risk.

- Hypnotics, narcotic agents and inhalation agents with balanced anesthesia usually minimize the potential problems in a perioperative period.

- It should be given attention for the position of the patient during anesthesia.

\subsubsection{The general anesthesia management in elderly patients}

Given that the vast majority of hip fracture surgeries are performed on elderly individuals, general anesthesia guidelines should be followed in geriatric patients. At first, the premedication to which the respiratory tract may be depressed should be avoided or the dose should be reduced.

When performing volume replacement, the cardiac load should be avoided and the mean arterial pressure should be preserved. Respiratory depression can be avoided by minimizing the opioid dose. The inotropic medicines can be necessary to treat hypotension. The preserve of renal function should also be supplied.

We must make sure that the neuromuscular block is completely turned. For postoperative analgesia, regional anesthesia techniques should be used and the dose should be adjusted.

\subsection{Regional anesthesia}

There is a long history for epidural and spinal block interventions and the use of these interventions for pain relief. Both techniques are widely used around the world. They have some advantages such as regional anesthesia interventions, early mobilization, postoperative pain relief and reduced risk of DVT, hypoxia and conscious problems [12]. There are a number of studies in geriatric patients that indicate the superiority of regional anesthesia in hip fracture operations.

Advantages of spinal anesthesia: 
- Short acting time

- Provide better muscular relaxant effect

- More effective analgesia

- Rarely serious respiratory depression

- Cost-effective

- Easy to perform

Disadvantages:

- Provide low dose and toxicity.

- Rarely shaking.

- Hypotension is more frequent and sudden onset.

- The incidence of post-spinal headache is high.

- Upper level of blockage may be changed and cannot be adjusted.

- Postoperative analgesia cannot be obtained.

- Duration of the effect cannot be extended.

Advantages of epidural anesthesia:

- The desired block level can be titrated with the catheter.

- The duration of anesthesia can be extended with added doses.

- It is possible to obtain postoperative analgesia [13].

- No headache.

- The effect time is long.

- The depth of the block may vary depending on the agent.

- Lower boundary may vary; sacral distribution is limited.

- High dose is required.

- Shaking is often present.

Combined spinal-epidural anesthesia:

- It provides both spinal and epidural advantages as mentioned above in abdominal, lower extremity surgery and birth analgesia.

- It is possible to complete an inadequate spinal anesthesia. 
- It provides longer analgesia with opioids and/or local anesthetics even in postoperative period.

- The incidence of headache due to postdural puncture is low, and it also provides autogenous blood injection to treat/protect headache due to incidental postdural puncture.

\subsection{Peripheral nerve blockage}

If neuro-axial blockage is contraindicated, fascia iliaca block, femoral nerve block and sciatic nerve block can be applied. Peripheral nerve blockage is particularly useful in tumor patients developing opioid intolerance and using anticoagulants.

Lumbosacral blocks and femoral and sciatic nerve blocks may be used in hip arthroplasty operations in geriatric patients. These blocks also provide postoperative pain treatment. Since peripheral nerve blocks do not have sympathetic blockade, sudden hypotension cannot occur.

Advantages:

- Protection of the patient's consciousness and unaffected respiration.

- Higher possibility of providing unilateral blockage.

- The respiratory system is not affected.

- The effect of them on central nervous system is minimal.

- Less sympathetic blockade than regional techniques.

The use of ultrasonography in peripheral blockage:

- The use of Stimuplex may not be needed, the number of punches is reduced, the dose is reduced, and the blockage application time is reduced. Vasculature may not be prevented, the duration of the blockage is not prolonged in adults, and the shortened neuropraxia does not decrease [14].

\subsection{Evaluation after operation in hip fracture surgery}

Decent postoperative evaluation and care after the surgery are important factors for the healing of the patient. Post-anesthetic care includes periodic follow-up, if necessary; the treatment of respiratory, cardiovascular and neuromuscular functions of the patient; and monitoring of blood level, body temperature, pain intensity, nausea, vomiting, drainage, bleeding and urinary output.

The respiratory system is followed by the pulse oximeter monitoring. It is useful for early detection of hypoxia. ASA recommends oxygen supplementation therapy at risk of hypoxemia, during transport and at collection facilities. Oxygen therapy for up to 24 hours is especially recommended in the elderly group at risk of myocardial ischemia undergoing the hip fracture surgery. 
Cardiovascular system: ECG and blood pressure monitor should be continued during waking and compilation. It should not be forgotten that arthroplasty patients are elderly and cardiovascular comorbid diseases can be found and the risk of postoperative cardiac complications is high. The most important factor affecting mortality in geriatric patients is the current co-morbidities. Increase in troponin I is important in postoperative follow-up due to myocardial ischemia.

Assessment of neuromuscular functions (NMF) is done by physical examination and neuromuscular block monitors.

Body heat: Preservation of normothermia and periodic body temperature measurements should be made to prevent hypothermia, and heaters should be used if necessary. Both anesthesia recovery and postoperative patient comfort are important. In the treatment of thyroid disease, agents such as low-dose meperidine and tramadol can also be used.

Thromboemboli: Prophylactic anticoagulants, early mobilization, intermittent pneumatic compression devices, compression socks and neuro-axial anesthesia can reduce thromboembolic complications. Continuation of acetyl salicylic acid therapy is recommended in elderly patients. The use of regional anesthesia and anticoagulation is still controversial.

Nausea and vomiting: Periodic control of nausea and vomiting is important to detect complications. In the treatment of nausea and vomiting, 5-HT3 antagonists, tranquilizers/neuroleptics, scopolamine, dexamethasone, antihistamines or metoclopramide may be used.

Renal functions: Urinary retention is a common complication. It is especially more common after epidural anesthesia. This complication does not occur in peripheral nerve blocks.

Cognitive changes: Cognitive changes after hip fracture operations are common in elderly patients. There are studies showing that short-term cognitive changes are less frequent after spinal anesthesia. However, there was no difference in the long term.

\section{Postoperative analgesia methods in hip fracture surgery}

Pain management in hip fracture operations is multimodal. The combined use of different effect mechanistic analgesics provides less pain relief resulting in less opioid use and less complications [15].

Inadequate postoperative pain treatment causes prolonged hospitalization and additional malignancy. Inadequate pain management leads to the chronicity of the pain and the decrease in the quality of life of the patient.

Intraoperative analgesic treatment from the preoperative epidural catheter ensures that the patient does not have pain at the end of the operation. The patient can return to daily activities more quickly. Patient-controlled analgesia (PCA) technique is frequently used in postoperative pain management. It can be administered via peripheral intravenous or epidural catheter. Peripheral nerve blocks can also be provided with PCA using catheter. 
Bupivacaine and opioid protocols. are mostly used in regionalized PCA. It should be noted that opioids administered by the epidural route may also cause sedation.

Periarticular injection and multimodality in patients with hip arthroplasty should be part of the treatment [16]. Periarticular injection solution includes; bupivacaine $0.5 \%$ 200-400 mg, morphine sulfate 4-10 mg, epinephrine $300 \mathrm{mcg}$, methylprednisolone $40 \mathrm{mg}$, cefuroxime $750 \mathrm{mg}$ and $0.9 \% \mathrm{NaCl}$. The total volume is $60 \mathrm{ml}$.

Periarticular injection is applied in anterior capsule, iliopsoas tendon and inseriosion before reduction. After reduction, it is applied in abductor, fascia lata, snovia, gluteus maximus and insercius, posterior capsules, short external rotators.

Duration of hospital stay and ambulance: The average length of hospital stay is 3 days in hip arthroplasty. After a hip fracture repair, the average time for elderly patients is 20 days. Although there are many studies advocating that neuro-axial anesthesia is an advantage in ambulance [17], there are studies advocating that the anesthesia route is not effective.

\section{Author details}

\section{Guldeniz Argun}

Address all correspondence to: guldargun@yahoo.com

Departmant of Anesthesiology and Reanimation, Ankara, Turkey

\section{References}

[1] Patterson BM, Healy JV, Cornell CN, et al. Cardiac arrest during hip arthroplasty with a cemented long-stem component. The Journal of Bone and Joint Surgery. American Volume. 1991;73A:271

[2] Ranawat CS, Beaver WB, Sharrock NE, et al: Effect of hypotensive epidural anesthesia on acetabular cement-bone fixation in total hip arthroplasty. The Journal of Bone and Joint Surgery. British Volume 1991;73B:779

[3] Thompson GE, Miller RD, Stewans WC, Murray WR. Hypotensive anesthesia for total hip arthroplasty: A study of blood loss and organ function (brain, heart, liver and kidney). Anesthesiology. 1978;48:91

[4] Vazeery AK, Lunde O. Controlled hypotension in hip joint surgery: An assessment of surgical haemorrhage during sodium nitroprusside infusion. Acta Orthopaedica Scandinavica. 1979;50:433

[5] Weber RS et al. Anemia and transfusions in patients undergoing surgery for cancer. Annals of Surgical Oncology. 2008;15:34-35 
[6] Modig J. The role of lumbar epidural anaesthesia as antithrombotic prophylaxis in total hip replacement. Acta Chirurgica Scandinavica. 1991;151:1721

[7] Davis FM, Laurenson VG, Gillespie WJ, et al. Leg blood flow during total hip replacement under spinal or general anaesthesia. Anaesthesia and Intensive Care. 1989;17:136

[8] Djelonah I, Lefevre G, Ozier Y, et al. Fat embolism in orthopedic surgery: Role of bone marrow fatty acid. Anesthesia and Analgesia. 1997;85:441

[9] Frestein GS: Etiology and pathogenesis of rheumatoid arthritis. In: Kelly WN, Harris ED Jr, Ruddy S, et al. Textbook of Rheumatology. 5th ed. Philadelphia: WB Saunders; 1997. p. 851

[10] Carli F, Halliday D. Continuous epidural blockade arrests the postoperative decrease in muscle protein fractional synthetic rate in surgical patients. Anaesthesiology. 1997;86:1033

[11] Dyson A, Henderson AM, Chamlley D, et al. An assessment of postoperative oxygen therapy in patients with fractured neck of femur. Anaesthesia and Intensive Care Medicine. 1988;16:405

[12] Williams-Russo P, Sharrock NE, Mattis S, et al. Cognitive effects after epidural vs general anaesthesia in older adults: A randomized trial. Journal of the American Medical Association. 1995;274:44

[13] Moiniche S et al. A qualitative and quantitative systematic review of pre-emptive analgesia for postoperative pain relief: The role of timing of analgesia. Anesthesiology. 2002;96(3):526-527

[14] Busoni P. Central or peripheral blocks? Techniques in Regional Anesthesia and Pain Management. 2002;6:95-98

[15] American Society of Anesthesiologist Task Force on Acute Pain Management. Practice guidelines for acute pain management in the perioperative setting: An updated report by the American Society of Anesthesiologist Task Force on acute pain management. Anesthesiology. 2012;116(2):248-273

[16] Lunn TH, Husted H, Solgaard S, Kristensen BB, Otte KS, Kjersgaard AG, Gaarn-Lansen L, Kehlet H. Intraoperative local infiltration analgesia for early analgesia after total hip arthroplasty: A randomized, double-blind, placebo controlled trial. Regional Anesthesia and Pain Medicine. 2011;36:424-429

[17] Lunn TH, Kristensen BB, Gaarn-Lansen L, Husted H, Kehlet H. Post-anaesthesia care unit stay after total hip and knee arthroplasty under spinal anaesthesia. Acta Anaesthesiologica Scandinavica. 2012;56:1139-1145 